\title{
Design and Practice for National Fitness Network Consultation System
}

\author{
Kefei Wang1, a ,Li Zhang ${ }^{2, b}$ \\ ${ }^{1}$ Luoyang Normal University Institute of P.E , Luoyang Normal University ,China \\ ${ }^{2}$ Mathematical Science College, Luoyang Normal University , China \\ akf36986@aliyun.com,bz198118@126.com
}

Keywords: national fitness; network consultation; design; practice

\begin{abstract}
According to national fitness knowledge and theory, combine module theory and Internet technology designed national fitness network consultation system. This paper makes explanation on design ideas, technology crucial, module and function design, working example of the system. This system can assess body functions,give health and body building knowledge treating plan, sport and body building prescription, provide Internet platform for all the sports participants scientific body training. So the system has important practice and promotion values.

National Physical Fitness and health is a state guarantee of continuous development, social development, physical level and an important indicator of the degree of civilization, is the key to our construction of sports power. Party and government have always attached great importance to people's health, the State Council in 1995 promulgated the "National Fitness Program" in 2009, promulgated the "National Health Bill", and continue to expand the work of the national physical testing are the people provide health protection. However, due to economic levels, social conditions, health awareness of the constraints in the implementation of regulations and outline the process there have been some problems, many people on too few knowledge about sports, they do not know how to exercise, not a scientific and rational exercise, affect training effect. Therefore, it is necessary to use network technology combined with the fitness guide, so that the masses for different fitness needs more convenient to search, consulting, to achieve a reasonable, effective, science and fitness purposes. To this end we have developed a "National Fitness Network Consulting System" (National Fitness Network Consultation System), hereinafter referred to as NFNCS system.
\end{abstract}

\section{Research Objects and Methods}

\subsection{Research Objects}

The research objects of NFNCS system include the fitness network resource library construction, network consulting system design and development, physical function evaluation, counseling and exercise prescription, system maintenance personnel and the services and consultants people from all walks of life.

\subsection{Research Methods}

\subsubsection{Literature Study}

The theoretical basis is established based on collecting, sorting, and analyzing texts of relevant information from books, newspapers, audio-visual documents and internet.
1.2.2 Survey and Interviews
a. network survey: survey through the internet
b. social survey: issuing questionnaire surveys to possible users 
c. interviews: interviewing specialists experts, scholars and professors

d. visits: members of the research team visiting different populations

\subsubsection{Network Design and Software Development}

The network and software of NFNCS system require the use of computer programming, and the development of appropriate network system and software. Its functions should concentrate on consultants' need, and meet the demand of consultation, function, technology and system, using the $\mathrm{C}++$ method, an object-oriented way, with Visual Studio VC6.0 and ASP 3.0 for development.

\subsubsection{Test Feedbacks}

When the NFNCS system is finished, we have tested it on sports administration and universities network, and widely soliciting opinions from users, sports, fitness experts and web developers to made some advices and improvement.

\section{Development of the NFNCS System}

NFNCS system design starting point is to solve our lack of fitness instructors, better to raise the masses of sports knowledge, training interests, to develop good exercise habits, to help them learn science and enhance the physical fitness, disease prevention promote the function of individual development. Build fitness network advisory system is to understand the needs of our national sports and physical status, based on data obtained in the field based on the Internet to establish a relatively complete fitness resource library and counseling, evaluation index system. The NFNCS system under the development platform of Visual Studio VC6.0 and ASP 3.0 have the following advantages. It is a program based on the universal language which can build a powerful web application program. It puts the program on the server, and compiles the program when the sever runs for the first time, thus improving the efficiency. The NFNCS system is very powerful and adaptive, for it is programmed in many kinds of languages, and now it supports C\#, VB and Jscript. The System makes such trivial tasks as questionnaire submission, client authentication, distribution system and configuration of the web sites much simpler. The System programming uses a character-based, grading configuration system, in which the configuration information is stored in a simple text, and it does not need the local administrator tools, making the server environment and application setting work more easily. It also takes it into consideration that administrators can define plug-in module in their own codes.

\subsection{Construction and Systematic Analysis of Resource Database}

On the basis of the relevant content and data analysis of fitness knowledge and guidance, combined with the international and domestic relevant research efforts, using object-oriented approach, the "class" and "object" of the network consultation and management system and its functions and interrelations have been researched. The resources database can be classified into such kinds as common knowledge and methods, functional level, movement-based, exercise goals, exercise programs, exercise prescription, case studies, under each of which there is a more specific second directory.

\subsection{Design and Development of the System}

Based on the collection and systematic analysis, the system modules have been reasonably arranged; and the "class" and "object" of the module have been refined; its operation and attribute, and state changes, the interaction between objects have been analyzed. The study focuses on how to establish a user-friendly and humanized operation system, on how to process data and models, on 
how to solve the technical problems in encoding data and how to solve the optimization of the database system, the scope of control, and data restoration and backup.

\subsection{Aalysis of Demand and Maintenance}

According to the analysis of system demand, including the demand of consultation, function and the system, the details of the system is studied. The system maintenance section is designed for system administrators to manage system, including the maintenance of knowledge base, the users' database. It also enables administrators to facilitate the conducts of the information retrieval, adding, modifying and deleting operation.

\subsection{System Deployment}

Using ASP.net, the research focuses on how to deploy the system of solutions, and share the resources on the internet..

\section{Key Technology of the NFNCS System}

A sound resource base should be established to ensure the correctness, progressiveness, systematization and integrity of the contents.For each consultant enter the system can accurately understand their function level, and fitness the way to retrieve, fitness knowledge, scientific and rational exercise prescription, fitness guide is a resource library focused on the construction.A bold and definite system operating interface makes consultation simple, friendly and humanized, which can also help each consultant easily learn how to use the system and make full use of the system features and resources. Meanwhile, according to the different needs of consultants, personalized features can be well-implemented.How to make the system truly scientific and effective to improve fitness effect is one of the key study.

\section{The Practice of the NFNCS System}

\subsection{The NFNCS System modules and its functions}

The NFNCS system is composed of the main module, nine second-level modules and many third-level modules. First, the consultant $\log$ in, $\log$ in to their detection in the national constitution of the main indicators to fill in the questionnaire, Include the general indicators (age, gender, etc.), body shape index (height, weight, chest, etc.), physical function indicators (pulse, lung capacity, etc.), physical indicators (Sit and Reach, grip strength, step test, sit-ups ). Questionnaire results contrast with the data in the database category, to identify the ratings of the consultant body. Then, fill out the required movement-based consultant, training purposes, medical history and other issues, the database system based consultation system, to the consultant to provide relevant knowledge and exercise prescription. Finally, the system can explain the exercise prescription and proposed notices of operation. Meanwhile, the health conditions and prescription effect can process real-time feedback and suggestions.

\subsubsection{Logging-in}

Logging-in module includes the logging-in of passers-by and members. Only after the legal logging-in, the system can be operated and used. The logging-in authority is different to different people. The system establishes sports Fitness records and conduct real-time health and body building instructions for members.

\subsubsection{Functional assessment}

The Functional assessment module includes questionnaires and evaluation module. The aim is 
to master the consultant's assessment of their level of physical condition, excluding contraindications sports, to provide the theoretical basis for scientific body building and formulate exercise prescription.First require consultants to fill in the data of the determination of the national constitution. The systems can analysis these datas,integrate resource library and the national constitution and health standard requirements, the subjects's relevant indicators should be converted to values and given total score, all part of the indicators sorted from high to low, given the most improved part and recommendations.

\subsubsection{Fitness knowledge and training goals}

To raise awareness of health knowledge, physical fitness evaluation and sports fitness knowledge and understanding designed the query module, everyone landing can consult their interested questions. Exercise target formulated according to different needs of the people and their levels. Consultants need to fill out the questionnaire, but also for provide the basis of issuance scientific exercise prescription.

4.1.4 Exercise prescription and means of implementation

It includes three modules of prescription background knowledge, exercise prescription, prescription Implementation considerations module, exercise prescription is the focus of these modules. it is sports and fitness method according to personal physical condition, medical history and exercise goals to take. The four elements of exercise prescription is:a reasonable exercise intensity, a reasonable content, a reasonable time and a reasonable exercise frequency. In the design of exercise prescription, the system will first determine these elements, and then selected content from the database, combined with the consultant's actual situation, consider the case condition of the individual's physical and movement-based, designed to exercise prescription meet the personal characteristics.Means of implementation is explanations and instructions to help advisors better understanding the exercise prescription, and give key considerations notes and tips to help advisors better operation exercise prescription to achieve the desired effect.

\subsubsection{Feedbacks and Suggestions}

It includes the effect Survey,prescriptions adjust and exercise tips.when the consultants log in again, the system will understand the consultant's situation and whether there are not suited. Then the system can monitor the fitness process and make necessary adjustments, to provide different tips and precautions at different stages.

\subsubsection{Exchanges and Help}

The system sets up network exchange platform and forums to promote the exchanges between consultants and relevant experts, professors and Sports Health Scienceteachers, and solves the problems online. Meanwhile, the system also provides helpful information of common problems and development.

\subsection{The running of the NFNCS system}

Through the running and practice of the NFNCS system, many experts, body building men in all walks of life $\log$ in this system, learn the Knowledge about the sports and fitness from consultation, scientific guidance their fitness activities,make helpful suggestions.It make us accumulated valuable experience. For example, at 3 p.m. on 2 May, in 2010, student named Liu Jia logged in the system consultation for fitness and weight loss. 


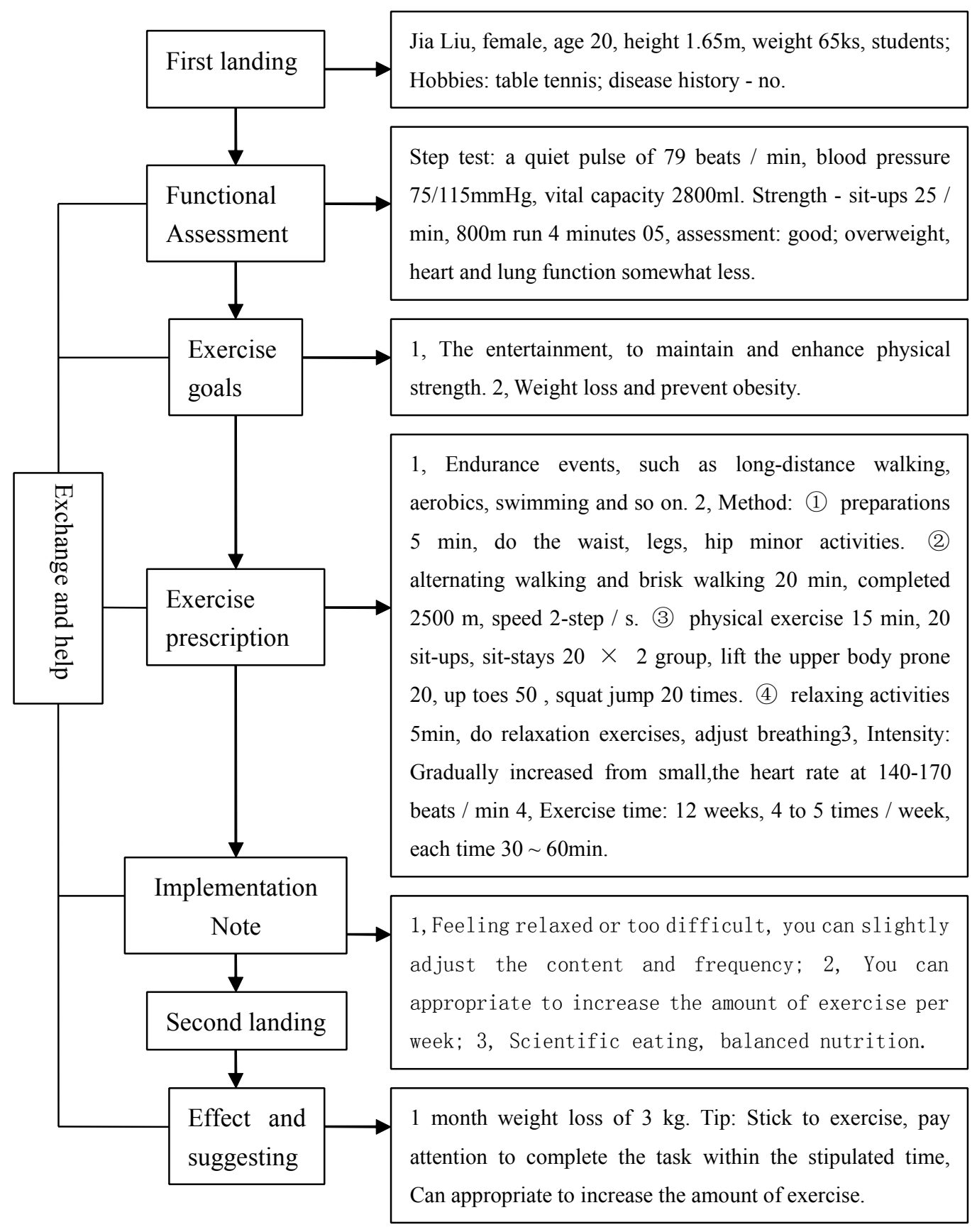

Fig. 1: Operation Process of the NFNCS System

\section{Conclusion}

The NFNCS system can guide the fitness for different populations, provide the appropriate health knowledge, methods, measures, issuing exercise prescription, make real-time advice and tips.The system designed new, simple and practical. The system answers consultant's questions and assessment physical function has the characteristics of convenient and accurate, fitness exercise prescription issued by scientific and effective, easy to operate, also can serviced for personal needs. In future, the standard mode of national Fitness consultation should be established; the network consultation resource database should be enriched; and the module design should be optimized to provide very convenient corresponding information for indicators. The system should established intelligent feedback mode, after a stage in the fitness, system should feedback the information to database,for individuals to build a comprehensive health and fitness file. Meanwhile, the NFNCS 
system should be integrated with network technology development, gradually expand the experimental range of the National Fitness consultation, broaden the channels of information feedback and exchanges, and continuously improve the structure and functions of the system.

\section{References}

[1] Cao Jie. Student Advisory System Fitness Network feasibility study [J]. Harbin Institute of Physical Education, 2009 (3) :37-40.

[2]Chiu-fun, Yao new. Sports team crisis counseling crisis intervention model of the system [J]. zhejiang Sports Sciences, 2008 (1) :24-27.

[3]Liang Jianxiu, Zhou Yanming. Students Advisory System Health Management and Practice [J]. Beijing Sport University, 2005 (10) :1372-1374.

[4]Peng Zehua. Network Consulting Information Library Organization and Management [J]. Information Theory and Practice, 2005 (2) :159-161.

[5]Zhao Jiang Hong, Liu Zhiqiang. Students individualized exercise prescription system design and computer consulting practice [J]. Xi'an Institute of Physical Education, 2005 (3) :118-121.

[6]Lu Liping, Xie Youchun. College Sports PC Expert System for the preliminary study [J]. Anqing Teachers College, 2002 (3) :92-94. 